\title{
EDITORIAL CONMEMORATIVA
}

Y casi sin darnos cuenta... ha pasado el tiempo: Cultura de los Cuidados cumple un cuarto de siglo

\section{And almost without realising it, time has passed: Culture of Care is a quarter of a century old.}

\section{Finalmente, quase sem perceber, o tempo passou: a Cultura dos Cuidados tem um quarto de século \\ José Siles}

Catedrático del departamento de enfermería. Facultad Ciencias de la Salud. Universidad de Alicante.

Orcid: https://orcid.org/0000-0003-3046-639X

Correspondencia: Departamento de enfermería. Facultad de Ciencias de la Salud. Universidad de Alicante, Alicante. CP 3080

Correo electrónico de contacto: jose.siles@ua.es

Cómo citar esta editorial: Siles, J. (2021). Y casi sin darnos cuenta... ha pasado el tiempo: Cultura de los Cuidados cumple un cuarto de siglo. Cultura de los Cuidados (Edición digital), 25(59). Recuperado de http://dx.doi.org/10.14198/cuid.2021.59.01

Recibido:25/11/2020 Aceptado: Invitación editorial

\section{RESUMEN}

En esta editorial se hace un repaso a la historia de la revista cultura de los cuidados. Se describen sus antecedentes, factores predisponentes, su origen y evolución a lo largo de un cuarto de siglo.

Palabras clave: cultura de los cuidados; historia de cultura de los cuidados; historia de las revistas de enfermería.

\section{ABSTRACT}

This editorial reviews the history of the culture of care publication. It describes its background, predisposing factors, its origin and evolution throughout a quarter of a century.

Keywords: culture care; history of culture care; history of nursing journals.

\section{RESUMO}

Este editorial revê a história da cultura da revista de cuidados. Descreve os seus antecedentes, factores predisponentes, a sua origem e evolução ao longo de um quarto de século. 
Palavras-chave: cultura de cuidados; história da cultura de cuidados; história das revistas de enfermagemn

Cultura de los Cuidados llega a su XXV aniversario casi sin darse cuenta, aunque comprobando que nadie es inmune al paso del tiempo y que éste deja siempre su poso, la huella de su paso absolutamente por todo: piedras, árboles, ríos y mares, animales, estrellas o planetas, personas y, por supuesto, revistas que tampoco se libran de este tránsito existencial. Los que estamos al pie del cañón o en la sala de calderas de este navío editorial, tal vez, somos los menos indicados para interpretar sin subjetividades, nostalgias o añoranzas esta circulación a través del tiempo. A estos posos o reliquias que atestiguan el paso del tiempo, Aróstegui los denomina "cambios” (Siles, 2011).

Sin lugar a dudas... se han producido muchos cambios desde el año 1996. Bastaría con echar una ojeada a nuestras mesas de trabajo actuales para percatarnos las enormes diferencias que existen respecto a aquel mismo mueble que ejercía idéntica función hace un cuarto de siglo: poblado de ordenadores enormemente gruesos y que rugían como fórmulas I, pero que iban tan lentos como el caballo del malo, montañas de cartas que poblaban las mesas dado que eran por entonces la principal forma de comunicación a distancia, algunas que otras láminas de acetatos con las que aún se diseñaban las transparencias a mano con rotuladores “ad hoc”, etcétera. Otra estética tecnológica muy diferente a la actual colmaba nuestro escenario laboral. Pero, poco a poco, casi sin darnos cuenta, todo esto ha ido cambiando y ya no recibimos ni enviamos cartas, nuestros ordenadores son sílfides silenciosas y ultra- rápidas y de los acetatos no queda ni la sombra.

En el contexto de la producción editorial también hemos asistido a esos cambios producidos por el paso del tiempo.
En aquellos años de mediados de los noventa, las revistas eran mayoritariamente una parte sustancial de la industria papelera donde la imprenta era un elemento esencial. Las dificultades en el diseño eran mayores porque tampoco estaban al alcance de la mano los programas que realmente servían para diseñar las publicaciones científicas y esto, naturalmente, repercutía en el encarecimiento de un producto cuya comercialización no sólo no era sencilla, sino que, como es el caso de Cultura de los Cuidados, ni siquiera entraba dentro de los planes del equipo editorial.

Como en todo nacimiento, había que designarle un nombre a la nueva publicación. La denominación de la revista fue muy meditada y esto hay que explicarlo, aunque sea brevemente. Finalmente se bautizó a la criatura de papel (entonces esta era su encarnadura), bajo el nombre "Cultura de los Cuidados". ¿Por qué esta denominación? Pues, simplemente porque sí que teníamos claro el objetivo de la revista. Para desentrañar el propósito primigenio que se perseguía con la revista es conveniente "pasearse" con detenimiento por el concepto de cultura y cuidado. Si tal como afirman algunos investigadores, la cultura constituye un crisol donde se funden comportamientos, valores, creencias, tradiciones, simbologías, significados y sentimientos que tienen, entre otras, la función de contribuir al proceso de satisfacción de necesidades del ser humano confiriendo a este mecanismo cierto sentido y cohesión social (Siles, 2010); estaba claro que el cuidado no era ajeno a ese acrisolamiento cultural y que, en cualquier contexto, los cuidados sólo adquirían sentido si estaban regulados culturalmente. En definitiva, el objetivo que orientó la creación de la revista estribaba en servir de plataforma donde se pudieran publicar artículos que temática y metodológicamente tuvieran en cuenta este carácter cultural e histórico de los cuidados. Siguiendo con el mismo planteamiento se vertebró la revista 
en cuatro grandes bloques temáticos: fenomenología, historia, antropología y teoría y método.

\section{Política, sociedad y cultura en España y el Mundo en 1997}

El acrisolado panorama en el que Cultura de los Cuidados vio la luz constituía una realidad en la que incidieron factores de toda índole: políticos, sociales, culturales, económicos, etc. En aquel año (1997) gobernaba en España el Partido Popular con José María Aznar como presidente del Gobierno. Se iniciaba un nuevo ciclo tras 14 años de gobierno del Partido Socialista Obrero Español. Otra novedad acontecida este año fue el abandono de Felipe González, sustituido como secretario general del partido por Joaquín Almunia. Fue el año en el que en España todavía ETA seguía asesinando y secuestrando (el secuestro de Ortega Lara o del posteriormente asesinado Miguel Ángel Blanco como muestra) mientras "Gesto por la Paz" reivindicaba la paz. Paralelamente, en el universo poético, el inolvidable Ángel González nos hablaba de "Las otras soledades de Antonio Machado" en su discurso de ingreso en la Real Academia Española (tomando el relevo del tristemente desaparecido antropólogo Julio Caro Baroja).

A nivel internacional, tal vez el acontecimiento más relevante (aunque siempre habrá escépticos que lo duden) radicó en el nacimiento de los dibujos animados de "Pokemon" hecho que provocó en Japón un fenómeno de salud poco común: centenares de niños fueron hospitalizados por ataques de epilepsia tras haber visto el episodio de Pókemon "Dennō Senshi Porygon” . Claro está que habría que considerar otros factores como el político y la economía: Bill Clinton -ya acuciado con el Monicagate- estaba en los inicios de su segundo mandato presidencial precediendo la fusión de dos gigantes bursátiles: Morgan Stanley y Dean Witter escenificando la que iba a ser la más imponente sociedad de valores de Wall Street. Simultáneamente a la irrupción de este fenómeno financiero, la sonda espacial Mars Pathfinder de la NASA se posó en la superficie de Marte. Mientras tanto, en Escocia, además de seguir fabricando whisky, en el farragoso terreno donde se desdibujan las fronteras entre la ciencia tradicional y la ciencia ficción, aparecía en escena la oveja clonada "Dolly", casi coincidiendo con la muerte en París, en un misterioso accidente automovilístico, la princesa Diana de Gales. En lo concerniente al aspecto demográfico, puede que sea significativo considerar un hecho que marca el fin de una tendencia y el inicio de otra: En Suecia, por primera vez desde principios del siglo XIX, fallecieron más suecos que los que nacieron.

$\mathrm{Al}$ otro lado del cuadrilatero geopolítico, Borís Nikoláyevich Yeltsin, recalcitrante acólito de la bebida nacional "Smirnoff" tomaba el relevo de Gorbachov, tal vez el político que más hizo por la caída del muro y que había cambiado el panorama de su país. En la India otro suceso trágico vuelve a reivindicar la "estulticia sin fronteras del ser humano" cuando otro grupo terrorista, autodenominado "Ranvir Sena" (integrado mayoritariamente por brahmanes ultraconservadores) asesinan a una decena de personas de "casta baja” y emplean su sangre como tinta para pintar el nombre del grupo en las paredes del pueblo.

Desde la perspectiva de las epidemias, los virus y las herramientas de contención respecto a los mismos, acaece a finales de año un hecho que hoy día está de rabiosa actualidad: El Gobierno de Hong Kong ordena matar todos los pollos para prevenir una nueva variante de gripe aviaria.

En el ámbito iberoamericano, acontece un fenómeno sorprendente que, empero, no despertó gran interés: el Congreso Nacional de Ecuador destituyó al presidente de la nación, el cantante y abogado Abdalá Bucaram, aduciendo el inquietante estado de su salud mental. En el 
mundo del cine se estrenan grandes producciones como: “Titanic”, "La vida es Bella”, “El indomable Will Hunting”, aunque los “Oscar" agraciaran como ganadora total a "El Paciente Inglés." Mientras, la película "El Che" vuelve a glosar el desembarco de Fidel Castro y el Che en Cuba, a la vez que en España “Brácula: Condemor”, con el desaparecido y añorado Chiquito de la Calzada, obtenía pingües beneficios de taquilla.

Situación de los estudios de enfermería en 1997 con especial atención a las iniciativas desarrolladas en la Universidad de Alicante

En el contexto educativo aún faltaban dos años para que se firmase la Declaración de Bolonia (1999) que daría el pistoletazo de salida a una compleja carrera cuya meta era -y sigue siendo- el proceso de convergencia europeo de educación superior. En lo concerniente a los estudios de enfermería, seguía vigente el plan de estudios de 1995 (Diplomatura) y en la universidad de Alicante se impartía Fundamentos de enfermería en tres módulos (Fundamentos histórico-antropológicos de enfermería I; Fundamentos teóricos y metodológicos de enfermería II; Fundamentos de enfermería en el sistema de necesidades y los cuidados básicos III). Asimismo, en el plan de estudios vigente en aquella época se contemplaban asignaturas que influyeron significativamente en la creación de la revista Cultura de los Cuidados: Pedagogía aplicada a los cuidados de enfermería e Historia de la enfermería en España. Eran momentos históricos de gran trascendencia para el futuro de la enfermería. Faltaban apenas un par de años para que se sancionara el título propio de segundo ciclo que rompería el "techo de cristal" de la enfermería: en abril de 1998 la Universidad de Alicante aprobó el título propio de segundo ciclo. Esto supuso un hito en la historia de la enfermería española, dado que era la primera iniciativa en España cuyo objetivo consistía en dotar a las enfermeras de la plena capacidad docente e investigadora, incidiendo de pleno durante el futuro inmediato, en la gestación de diversos proyectos en este sentido: máster y doctorados.

La historia es una ciencia hermenéutica y sería imposible describir todo lo anteriormente mencionado sin considerar a interpretación hechos que, lejos de reflejar meras anécdotas personalescomo el enfrentamiento que en 1996 protagonizaron dos cartageneros que, a la sazón, gobernaban los rumbos, por un lado, de la Comunidad Valenciana (Eduardo Zaplana, Presidente de dicha comunidad) y, por otro, de la Universidad de Alicante (Andrés Pedreño, Rector de la Universidad de Alicante). Esté enfrentamiento pudo incidir en la segregación de la Facultad de Medicina de la Universidad de Alicante y su integración en otra de nueva creación: la Universidad Miguel Hernández de Elche. En este contexto, la Universidad de Alicante se quedaba huérfana en materia de Ciencias de la Salud y, por ello, es probable que todo este guirigay rebasara el nivel de simple chascarrillo y terminara influyendo, junto con otros factores, en el auge de la enfermería en la Universidad de Alicante (Siles, 2011) .

\section{Cuidados}

Origen de la revista Cultura de los

A poco que se eche la vista atrás, resulta sencillo comprobar que existían ya unas condiciones previas al surgimiento de la revista, antecedentes que denotaban la efervescencia de un momento candente de álgida creatividad, donde imperaba el espíritu colaborativo e ilusionante entre los estudiantes de la diplomatura y los docentes del departamento de enfermería que tuvieron aquella estrambótica iniciativa.

La implantación de El Seminario de Historia y Antropología de los Cuidados en 
1996, supuso un paso preliminar que desembocó en la creación de la Asociación Nacional de Historia y Antropología de los Cuidados (1998), una plataforma que sirvió de base para que hicieran reacción diversas sinergias: la asignatura pedagogía aplicada a los cuidados, Foro de Enfermería para el desarrollo, doce ediciones del premio Vida y Salud de narrativa (Siles, 2019), ocho Jornadas Internacionales de Cultura de los Cuidados, y diferentes asignaturas de grado y posgrado: las asignaturas Cultura de los Cuidados (diplomatura), Historia y Cultura de los Cuidados (máster), el máster oficial Cultura de los Cuidados y, por supuesto, el programa de doctorado Enfermería y Cultura de los Cuidados.

La revista surgió en medio de un panorama colaborativo entre distintas iniciativas de la disciplina enfermera, que compartían la urgencia de superar el paradigma neopositivista y recuperar para la enfermería una perspectiva epistemológica mucho más centrada en la realidad antropológica, fenomenológica, histórica y social de los cuidados. En este contexto de cooperación, que sirvió para unir a diferentes personas y grupos que compartían una visión de la enfermería, destacaron iniciativas como: La Fundación Index con Manuel Amezcua a la cabeza y la revista del mismo nombre, el Seminario de Historia de la Enfermería liderado por Francisca Hernández Martín, el Portal Híades y la revista del mismo nombre tutelados por los hermanos García Martínez, etc.

Uno de los eventos más distintivos de aquella atmósfera cooperativa que caracterizó la evolución de esta pléyade de iniciativas que orbitaron alrededor de Cultura de los Cuidados, fue, sin duda, la organización de las III Jornadas Internacionales de Cultura de los Cuidados que sirvieron para celebrar conjuntamente con la Transcultural Nursing Society su trigésima reunión anual y con la Fundación Index, la VI reunión sobre investigación cualitativa en salud . Hasta la fecha, las últimas jornadas celebradas han estado dedicadas monotemáticamente a los cuidados de salud de la mujer en el contexto de la mutilación genital femenina (dentro del marco de la violencia de género). Así las VIII Jornadas internacionales de cultura de los cuidados: Violencia de género, mutilación genital femenina y cuidados de salud celebradas en Alicante en Junio de 2019 contaron con la aportación de prestigiosos expertos en la materia como: Adriana Kaplan, coordinadora del Grupo Interdisciplinar para la Prevención y el Estudio de las Prácticas Tradicionales Perjudiciales (UAB), y de mujeres que, habiendo sufrido tal práctica, se han transformado en líderes que trabajan por su erradicación: Asha Ismail (Save Generation) y Fátima Djarra (Medicos del Mundo) (Siles, 2018).

Volviendo a los inicios de la revista, a pesar de que era difícil vaticinar que un proyecto así durara lo suficiente como para estar escribiendo esta editorial conmemorativa del cuarto de siglo de vida; lo cierto es que la consistencia de la idea de partida ha permitido la conservación de la estructura originaria de la revista en cuatro grandes bloques temáticos -además de las "Cartas al director" y la sección "Miscelánea”, dedicados a los cuidados y potenciando la perspectiva cualitativa de la disciplina: historia, fenomenología, antropología y teoría y método.

Así las cosas, el primer número de la revista vio la luz en Junio de 1997. Este parto fue posible sólo gracias a la confianza que mostraron en "la criatura" eminentes investigadores de la enfermería española de la época que, en un acto de generosidad que les honra, pusieron a disposición de una publicación sin bagaje previo ni alcurnia editorial alguna, trabajos cuya excelencia resultó determinante para que Cultura de los Cuidados comenzara a brillar con luz propia desde su primer número: Francisco Herrera, 
Revista científica de la Asociación de Historia y Antropología de los Cuidados (Universidad de Alicante)

Manuel Amezcua, Francisco Ventosa, Rafael Pascual, etc., pero en el número fundacional también se contó con una aportación extraordinaria que no provenía de la enfermería, sino de la literatura: Enrique Cerdán Tato nos "regaló” para la sección "fenomenología” un relato autobiográfico titulado "El gotero" en el que describía sus vivencias hospitalarias.

A lo largo de los años de recorrido de esta publicación con una abierta vocación armonizadora de las dimensiones humanísticas y científicas de la disciplina enfermera, han colaborado cientos de autores de diferentes países (Brasil, Colombia, Méjico, Ecuador, Chile, Uruguay, India, Argentina, Perú, Salvador, Honduras, Venezuela, España, Francia, Suiza, Portugal, Estados Unidos, etc.), y las visitas a los diferentes artículos han tenido decenas de miles de descargas, estando indizada en diferentes bases de datos $\mathrm{y}$ repertorios científicos: SCOPUS, Emerging Sources Citation Index (ESCI), CINAHL, CSIC, REDIB, DOAJ, LATINDEX, MIAR, DIALNET, GOOGLE SCHOLAR, etc.

Desde el principio contamos, como ya se ha señalado, con la colaboración de autores que son los que han logrado que hoy día la revista haya alcanzado el prestigio y el lugar que le corresponde en la amplia y variada topografía editorial de las ciencias de la salud, ocupando un lugar significativo en la temática histórico-antropológica e investigación cualitativa de los cuidados. Además de los investigadores mencionados anteriormente, Cultura de los Cuidados ha tenido el honor de contar desde los inicios con las aportaciones originales de prestigiosos científicos de la disciplina enfermera en el ámbito iberoamericano como: Carmen de la Cuesta, Francisca Hernández Martín, Ana Piera, Antonio Claret García Martínez y Manuel Jesús García Martínez, Genival Feranandes, Taka Oguisso, Mercedes Fraile, María Elisa
Moreno-Fergusson, Teresa Miralles, Fernando Porto, Bárbara Barrionuevo Bonini, Ximena Isla, Carlos Lousada, Almerinda Moreira, Alfonso García Hernández, Cristo Marrero y, en fin, un largo etcétera que haría esta lista interminable. A todos ellos, tanto a los mencionados como a los omitidos por la fuerza de las circunstancias, queremos darles nuestro más sincero agradecimiento.

En el contexto anglosajón tenemos también que destacar autores que nos han hecho crecer con sus colaboraciones originales: La misma Madelaine Leininger (fundadora de la enfermería transcultural), quien ya nos obsequió con una editorial cuando la revista aún estaba dando los primeros balbuceos (Leininger, 1999, 2004); Marjory Gordon, creadora de los patrones funcionales de salud, primera presidenta de la NANDA y declarada leyenda viva de la enfermería por la Academia Americana de Enfermería (Gordon, 2000); Larry Purnell, que gestó y desarrolló el modelo de competencia cultural (Purnell, 1999); Rachel Spector, creadora del modelo de herencia cultural, especialmente vinculada al programa de doctorado Cultura de los Cuidados y que nos honró con varias aportaciones (Spector, 2000, 2001, 2002, 2016); Callista Roy que desarrollo el modelo de adaptación que lleva su nombre y que fue declarada en el año 2007 “ Leyenda Viva”, al igual que Gordon, por la Academia Americana de Enfermería (Roy, 2000); y Dula Pacquiao, experta en enfermería transcultural que publicó sus investigaciones acerca de los ritos de paso que caracterizan culturalmente la homosexualidad (Pacquiao, 2000). Especial mención queremos hacer en esta editorial conmemorativa del cuarto de siglo a los autores que nos han dejado, como los tristemente fallecidos Carmen Chamizo Vega, Marjory Gordon, Antonio Galindo Casero, Alfredo Bermúdez González y Madelaine Leininger. 
Recapitulado sobre todo lo expuesto, sólo nos queda reiterar nuestro más sincero agradecimiento, además de los autores y lectores, a todos los que en las diferentes épocas de la revista, han colaborado entregando una parte importante de su tiempo al mantenimiento de esta empresa editorial desde diferentes roles: consejo editorial, científico y de redacción, revisores, becarios, etc. Gracias a todos.

\section{BIBLIOGRAFÍA}

Ferrer,E. (2006), Cultura de los Cuidados. Un recorrido de diez años. Cultura de los Cuidados, 10(20), 7-13. Recuperado de https://rua.ua.es/dspace/bitstream/10045/85 8/1/culturacuidados_20_1.pdf

Gordon, M. (2000). Pasado, presente y futuro de los diagnósticos de enfermería Cultura de los Cuidados. 4(7-8), 128-138. Recuperado de https://rua.ua.es/dspace/bitstream/10045/51 16/1/CC_07-08_16.pdf

Leininger, M. (1999). Cuidar a los que son de culturas diferentes requiere el conocimiento y las aptitudes de la enfermería transcultural. Cultura de los Cuidados, 3(6), 5-12. Recuperado de https://rua.ua.es/dspace/bitstream/10045/51 26/1/CC_06_01.pdf

Leininger, M. (2004). That the cultural needs of the people in the world may be met by nurses prepared in transcultural nursing. Cultura de los Cuidados, 8(16), 5-6. Recuperado de https://rua.ua.es/dspace/bitstream/10045/10 15/1/culturacuidados_16_01.pdf

Navarro, S. (1996). El rector de la Universidad de Alicante impide a Zaplana presidir la inauguración del curso. El País 6 Octubre.

Recuperado https://elpais.com/diario/1996/10/05/socied ad/844466434_850215.html

Pacquiao, D., \& Carney, M.K. (2000). La cultura de la homosexualidad: lecciones desde los ritos de «pasaje». Cultura de los Cuidados, 4(7-8), 75-86. Recuperado de https://rua.ua.es/dspace/bitstream/10045/50 88/1/CC_07-08_10a.pdf

Purnell, L. (1999). El modelo de competencia cultural de Purnell: descripción y uso en la práctica, educación, administración e investigación. Cultura de los Cuidados, 3(6), 91-102. Recuperado de https://culturacuidados.ua.es/article/view/19 99-n6-el-modelo-de-competencia-culturalde-purnell-descripcion-y-uso-en-lapractica-educacion-administracion-einvestigacion

Roy, C. (2000). El modelo de adaptación de roy en el contexto de los modelos de enfermería, con ejemplos de aplicación y dificultades. Cultura de los Cuidados, 4(7-8), 239-159. Recuperado de https://culturacuidados.ua.es/article/view/20 00-n7-8-el-modelo-de-adaptacion-de-royen-el-contexto-de-los-modelos-deenfermeria-con-ejemplos-de-aplicacion-ydificultades

Siles, J. (2010). Historia cultural de enfermería: reflexión epistemológica y metodológica. Avances de Enfermería, 18, 107-115. Recuperado de https://www.researchgate.net/publication/23 4045194_Historia_cultural_de_enfermeria_ reflexionepistemologica_y_metodologica

Siles, J. (2011). Historia de la enfermería. Madrid: DAE.

Siles, J. (2019). Fallo Vida y Salud de Relatos. Cultura Cuidados, 23(54): 429$430 . \quad$ Recuperado de https://rua.ua.es/dspace/bitstream/10045/96 352/8/CultCuid_54.pdf

Siles, J. (2018). VIII Jornadas Internacionales de Cultura de los Cuidados: 
Violencia de género, mutilación genital femenina y cuidados de salud (2019). Cultura de los Cuidados (Edición digital), 22(52). Recuperado de http://dx.doi.org/10.14198/cuid.2018.52.26.

Spector, R. (2000). La enfermería transcultural: pasado, presente y futuro. Cultura de los Cuidados, 4(7-8), 116-127. Recuperado

de https://rua.ua.es/dspace/bitstream/10045/51 15/1/CC_07-08_15.pdf

Spector, R. (2001) Heritage assessment. Cultura de los Cuidados, 5(9), 71-81. Recuperado de http://rua.ua.es/dspace/handle/10045/5076

Spector, R. (2002). Enfermería de cuidados culturales. Cultura de los Cuidados, 6(11), 5-10. Recuperado de https://rua.ua.es/dspace/bitstream/10045/48 57/1/CC_11_01.pdf

Spector, R.E. (2016). Cultural Competence. Cultura de los Cuidados (Edición digital) 20, 44. Disponible en: http://dx.doi.org/10.14198/cuid.2016.44.01

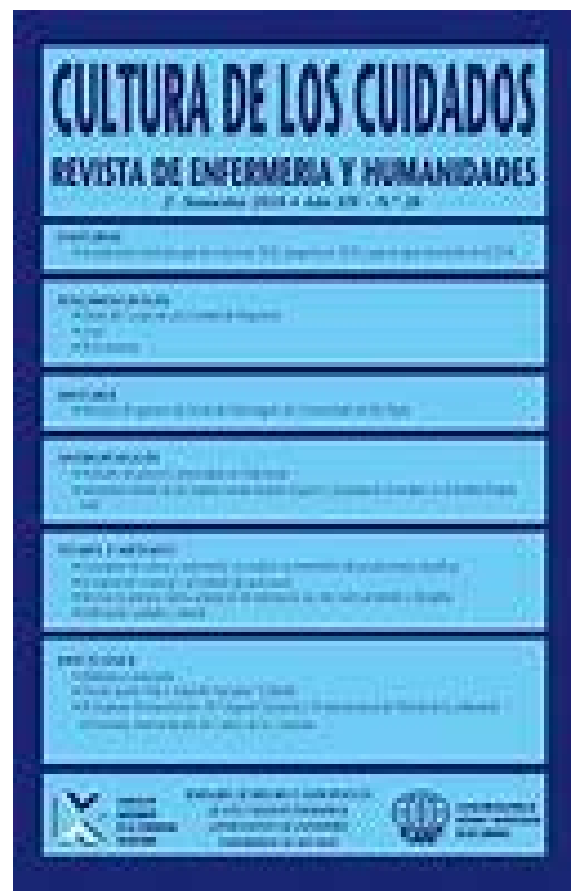

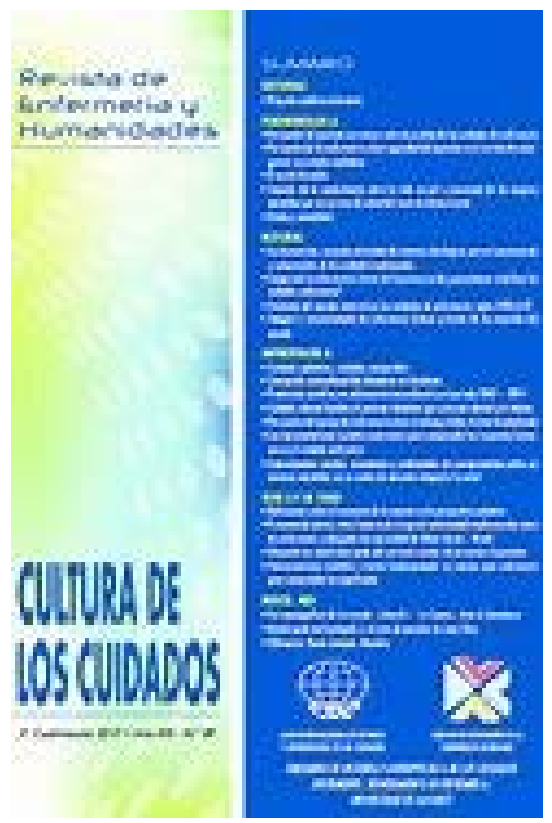

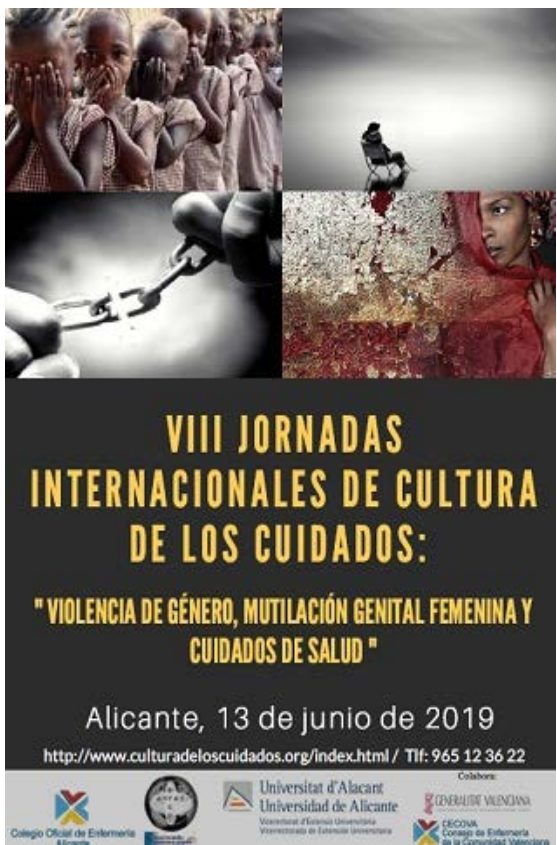

